\title{
Exploración del Comportamiento de un Instrumento para Evaluar la Función de Tutoría Académica en el Contexto de Escuelas Normales
}

\section{Exploration of the Behavior of an Instrument to Evaluate the Role of Academic Tutoring in the Context of Teacher Training School}

\author{
Salvador Ponce Ceballos * \\ Yaralin Aceves Villanueva \\ Brenda Imelda Boroel Cervantes \\ Universidad Autónoma de Baja California, México
}

\begin{abstract}
El presente trabajo documenta el proceso y resultados de un ejercicio de investigación de enfoque cuantitativo de tipo exploratorio, sobre el comportamiento métrico de un cuestionario para la evaluación de tutores universitarios, aplicado en el contexto de una Escuela Normal. El trabajo se apoyó en un cuestionario que evalúa el desempeño de tutores universitarios bajo el modelo de acompañamiento de estudiantes, integrado por treinta ítems derivados de tres dimensiones y cinco competencias. Dicho instrumento ha sido validado y probado en distintos contextos de formación universitaria. Los resultados se orientan a un buen comportamiento de los ítems para la evaluación de los tutores de Escuelas Normales ya que la prueba presentó un nivel de ajuste al modelo, por ítems y por categorías de respuesta. Parte de las conclusiones apuntan a la importancia de seguir explorando la evaluación de la tutoría para la mejora de los procesos de acompañamiento de estudiantes y la formación de los tutores académicos, de igual forma realizar más estudios métricos del comportamiento de los ítems para mejorar el proceso de evaluación.
\end{abstract}

Palabras clave: Tutoría; Evaluación; Escuelas de magisterio; Educación superior; Cuestionarios.

This paper documents the process and results of an exploratory quantitative approach research exercise on the metric behavior of a questionnaire for the evaluation of university tutors applied in the context of a teacher training school.The work was supported by a questionnaire that evaluates the performance of university tutors under the student accompaniment model, composed of thirty items derived from three dimensions and five competencies. This instrument has been validated and tested in different Mexican higher education context. The results are oriented to a good behavior of the items for the evaluation of the tutors of teacher training school since the test presented a level of adjustment to the model, by items and by response categories. Part of the conclusions point to the importance of continuing to explore the evaluation of tutoring for the improvement of student accompaniment processes and the training of academic tutors, also perform more metric studies of the behavior of the items to improve the evaluation process.

Keywords: Tutoring; Evaluation; Teacher training school; Higher education; Questionnaire.

*Contacto: ponce@uabc.edu.mx

issn: $1989-0397$

www.rinace.net/riee/

https://revistas.uam.es/riee
Recibido:

30 de noviembre de 2019

$1{ }^{\text {a }}$ Evaluación: 4 de febrero de 2020

$2^{a}$ Evaluación: 19 de febrero de 2020

Aceptado: $\quad 3$ de marzo de 2020 


\section{Introducción}

La Asociación Nacional de Universidades e Instituciones de Educación Superior (ANUIES) propuso, en el año 2000, un conjunto de líneas estratégicas de desarrollo que forjaban el futuro del Sistema de Educación Superior (SES); posteriormente generó diferentes programas, siendo uno de ellos el denominado "Desarrollo Integral de los Alumnos", que tenía como fin proporcionar apoyo a los alumnos por medio de programas de tutorías y desarrollo integral (Romo, 2011).

La ANUIES define a la tutoría como un proceso de acompañamiento cuyo objetivo es "proveer orientación sistemática al estudiante, desplegada a lo largo del proceso formativo; desarrollar una gran capacidad para enriquecer la práctica educativa y estimular las potencialidades para el aprendizaje y el desempeño profesional de sus actores: los profesores y los alumnos" (ANUIES, 2001, p. 30).

El modelo de tutorías planteado por la ANUIES consiste en que los académicos brinden una atención, ya sea de forma individual o en grupos pequeños a un número de alumnos asignados a su tutela, con el propósito de orientarlos, darles seguimiento y apoyo en su desarrollo cognitivo, social y personal; buscando que sea un proceso formativo y no solo informativo para que culminen su proyecto académico y disminuir así los índices de rezago y/o deserción (Romo, 2010).

En la propuesta de la ANUIES se plantea la tutoría como un apoyo complementario a la docencia con la intención de elevar la calidad y eficiencia terminal del estudiantado. Asimismo, se describen las temáticas en las que debe girar la capacitación para que un docente pueda fungir como tutor (Saavedra, Alatorre y Tremillo, 2015).

Este modelo de tutoría es una estrategia que se ha utilizado mayormente en las universidades asociadas a la ANUIES y paulatinamente en otras Instituciones de Educación Superior y Media Superior, como es el caso de las Escuelas Normales. Es de destacarse que, hasta el 2012, la tutoría (desde el enfoque de la ANUIES) se hace oficial en los planes de estudios de educación normal, quedando estipulado en el acuerdo 649 y 650 del Diario Oficial de la Federación, sin dejar de reconocer que seguramente este tipo de estrategias ya se utilizaban sin establecerse oficialmente en los planes de estudio como lo destacan algunos autores.

El presente artículo documenta los resultados métricos de la aplicación, en una Escuela Normal, de un cuestionario para la evaluación de tutores universitarios, el cual ha sido probado y validado en distintos contextos. En particular, la intención del presente ejercicio investigativo fue valorar su comportamiento en dicha institución. Asimismo, se busca presentar un panorama general sobre la incorporación de las tutorías académicas en este tipo de instituciones.

\section{Fundamentación teórica}

\subsection{Conceptos}

Para abordar la temática "Exploración del comportamiento de un instrumento para evaluar la función de tutoría académica en el contexto de Escuelas Normales" es preciso iniciar definiendo los conceptos fundamentales relacionados con el proceso de este 
programa de acompañamiento en la Educación Superior. A continuación, se presentan las definiciones de tutoría, tutor, tutorado, orientación y canalización.

\subsubsection{Tutoría}

Álvarez y Bisquerra (1996, citado por Redondo et al., 2012), definen la tutoría como una tarea que realizan los profesores, en el que a través de ella orientan a los alumnos sobre información académica y profesional, así como aspectos que impactan en su desarrollo personal, favoreciendo así la formación integral del estudiante.

A su vez, la UNESCO la define como el "conjunto de actividades que propician situaciones de aprendizaje y apoyan el buen desarrollo del proceso académico, con el fin de que los estudiantes orientados y motivados desarrollen automáticamente su propio proceso" (Arnaiz e Isús, 1998, citado por Romo, 2011, p. 48).

Posteriormente, la Asociación Nacional de Universidades e Instituciones de Educación Superior (ANUIES) definió a la tutoría como:

Un proceso de acompañamiento durante la formación de los estudiantes, que se concreta mediante la atención personalizada a un alumno o a un grupo reducido de alumnos, por parte de académicos competentes y formados para esta función, apoyándose conceptualmente en las teorías del aprendizaje más que en las de la enseñanza. (Romo, 2010, p. 51)

\subsubsection{Tutor}

Fernández (1991, en Redondo et al., 2012) define al tutor como la persona que dentro de la comunidad escolar engloba y se responsabiliza de guiar la evolución del alumno en lo que atañe a su aprendizaje y evolución personal, sirviendo de cauce a las intervenciones de las personas implicadas en la educación de los alumnos, con unas funciones que le son propias (p. 213).

Por su parte, Giner y Puigardeu (2008) lo definen como un coordinador de las experiencias de aprendizaje y de desarrollo integral, un mediador entre la universidad y el alumno y un agente de referencia en su mundo profesional (p. 24, citado por Gaitán, 2013).

De la Cruz, Chehaybares y Abreu (2011) definieron al tutor como un profesional con experiencia, dispuesto a compartir su conocimiento y dar consejos para facilitar el desarrollo intelectual, académico, personal o profesional de un aprendiz.

\subsubsection{Tutorado}

De la Cruz, Chehaybares y Abreu (2011) señalan que la literatura cataloga al tutorado como un novato, un aprendiz, alguien menos experto que el tutor. En este mismo tenor, Ramos y González (2011) lo definen como "una entidad que recibe la tutoría. [Alguien que] asimila y procesa información para adquirir conocimiento sobre su entorno y, entonces, ejecutar una operación bajo la supervisión del tutor; dicha acción no se ve limitada por los propósitos del tutor" (p. 225).

\subsubsection{Orientación}

Se entiende que orientar es ampliar el marco de experiencias, intereses, expectativas y oportunidades, permitiendo que el alumnado desarrolle las capacidades apropiadas para el trabajo autónomo, adquiera las habilidades polivalentes que se requieren para hacer frente a la resolución de problemas, reconozca la diversidad de alternativas posibles y pueda valorar distintas fuentes de datos antes de decantarse de forma consciente y 
autónoma por aquellas que considere más adecuadas y relevantes (Álvarez, 2005; Arias et al., 2005, citado por Álvarez y González, 2009, p. 74).

En este sentido, la ANUIES (2001) indica que uno de los objetivos específicos del sistema tutorial es la orientación, refiriéndose a ello como el apoyar al alumno en los problemas escolares y/o personales que se le presenten durante el proceso formativo, así como proporcionar la información pertinente que permita a los tutorados tomar decisiones académicas, el uso apropiado de los servicios y apoyos institucionales, así como la realización de trámites y procedimientos necesarios.

\subsubsection{Canalización}

La ANUIES (2001) indica que parte de los objetivos específicos de la tutoría es derivar a los tutorados a instancias especializadas para su atención en caso de dificultades en el aprendizaje, situaciones especiales como discapacidad, problemáticas personales, familiares, etc. La UABC (2012) entiende por canalizar en el ámbito de la Tutoría a "Encauzar al tutorado a los servicios de apoyo institucional, que den respuesta a sus necesidades y aspiraciones específicas" (p. 12).

\subsection{Las tutorías académicas en las Escuelas Normales}

Posterior a la incorporación de las Escuelas Normales al Sistema de Educación Superior Mexicano (Jiménez y Villafuerte, 2011), y como respuesta a la demanda del Plan Estatal de Fortalecimiento de la Educación Normal (PEFEN), en el 2005 inició la estrategia de tutoría en dichas instituciones (Mapén, Pérez Cupil y López Iglesia, 2016; Ramírez, Campos y Rodríguez, 2013). Sin embargo, como todo proyecto inicial, su instrumentación fue paulatina, heterogénea y no en la totalidad de las Escuelas Normales.

En los planes de estudios para la Formación de Maestros en Educación Primaria y en Educación Preescolar de 2012 queda plasmada la flexibilidad curricular y con ella las estrategias de asesoría y tutoría con el propósito de favorecer la trayectoria de formación de los alumnos, contemplando aspectos de su desarrollo profesional y formación integral (Diario Oficial de la Federación, 2012a, 2012b).

En este mismo tenor, la acción tutorial en las Escuelas Normales "consiste en un proceso de acompañamiento durante la formación profesional, que se concreta en la atención personalizada de manera individual o a un grupo reducido, por parte de académicos competentes y formados para esta función (...)" (DGESPE, s.f). Tiene como objetivo "orientar y dar seguimiento al desarrollo de los estudiantes, lo mismo que apoyarlos en los aspectos cognitivos y afectivos del aprendizaje. Asimismo, fomentará su capacidad crítica y rendimiento académico para incidir positivamente en su evolución social y personal (...)" (DGESPE, s.f).

Como puede observarse, tanto las funciones y actividades del tutor en las Escuelas Normales van acorde al modelo tutorial propuesto por la ANUIES. No obstante, con el paso del tiempo, las experiencias vividas en las Escuelas Normales constataron que, tanto el propósito de la tutoría, la forma de implementarla, así como el perfil necesario para fungir como tutor en este tipo de instituciones, varía en relación al resto de las IES, por lo que se llegó a la conclusión de sugerir una adaptación del modelo propuesto por la ANUIES a las realidades que viven las Escuelas Normales (Saavedra, Alatorre y Tremillo, 2015). 


\subsection{Posible confusión del uso del término tutoría en las Escuelas Normales.}

En los planes de estudio ya comentados, en el octavo semestre se ubica un espacio curricular denominado trabajo de titulación, en el que el estudiante normalista desarrolla actividades orientadas a la elaboración de su portafolio, informe de prácticas o tesis de investigación, para lo cual recibirá asesoría por parte de un docente. Asimismo, en este semestre se realiza la práctica profesional en una institución educativa del nivel de formación en donde el alumno es acompañado por un profesor (DGESPE, s.f.).

En este sentido, los lineamientos para organizar el proceso de titulación de los alumnos normalistas (SEP, 2012), contemplan la figura del asesor. Valenzuela y Herrera (2016) a su vez, puntualizan que “(...) el profesor-tutor viene a formar parte importante en la construcción del trabajo de investigación del futuro profesional de la educación (...)” (p. 8).

Las tutorías, las asesorías y el acompañamiento de prácticas se contemplan como estrategias de "acompañamiento académico y apoyo en la toma de decisiones $\mathrm{y}$, eventualmente, para la solución de problemas personales del estudiante" (Diario Oficial de la Federación, 2012a, 2012b). Con base en lo anterior, aunque los roles están claramente especificados, se infiere que, en la práctica, puede existir confusión, asumiendo al rol del tutor en las Escuelas Normales también funciones de "asesor" y “acompañante de prácticas” (cuadro 1).

Cuadro 1. Posibles funciones de los tutores en Escuelas Normales FUNCIONES IDENTIFICADAS PARA UN TUTOR EN ESCUELAS NORMALES

Acompañamiento
Académico desde el modelo de ANUIES.

Fuente: Elaboración propia.

\subsection{La importancia de la evaluación de los tutores en escuelas normales}

Para contribuir a la mejora de las estrategias de tutorías en las instituciones, es necesaria la evaluación permanente de los distintos participantes y la forma en que se están desarrollando dichas estrategias (De la Cruz, Díaz-Barriga y Abreu, 2010; Pérez Cusco, Martínez Clarés y Martínez Juárez, 2015; Zazueta y Herrera, 2008).

Los programas de tutorías pueden evaluarse a través del seguimiento de la trayectoria escolar de los alumnos y los indicadores de deserción y eficiencia terminal (ANUIES, 2001); la auto reflexión de los tutores sobre su desempeño para crear conciencia y reflexión en el mismo sobre sus fortalezas y debilidades, en relación a esta tarea y lograr así la mejora continua (Minor, Castellanos y Gómez, 2016), así como la percepción de los alumnos sobre la satisfacción (González, Carmona y Rivas 2007, en Pérez Cusco, Martínez Clarés y Martínez Juárez, 2015; Mapén, Pérez Cupil y López Iglesia, 2016).

El programa de tutoría en las Escuelas Normales es una de las estrategias para abordar las áreas de oportunidad de los estudiantes y lograr así el propósito educativo (Ramírez, et al., 2013). Al ser el tutor un actor principal en el proceso de tutoría es importante que se considere su evaluación con la intención de tener información sobre su desempeño, pero sobre todo para conocer la realidad que se vive en los procesos tutoriales que este emprende; considerando que el proceso de la evaluación permite identificar las fortalezas y áreas de oportunidad, así como corroborar si se está logrando el propósito del mismo. 
Lo anterior resulta un elemento clave para su buen funcionamiento. Sin embargo, la ANUIES (2010) refiere que son pocos los estudios realizados en torno a la evaluación de la tutoría.

Las Instituciones de Educación Superior han establecido sus propias propuestas de evaluación asociadas a sus realidades, condiciones e intenciones (García et al., 2016). Sin embargo, en el caso de las Escuelas Normales es limitada la evidencia sobre la evaluación de los tutores, posiblemente debido a su reciente incorporación como estrategia oficial en dichas instituciones (Ponce, Aviña, García y Caso, 2018). Por ello, es pertinente identificar las propuestas existentes que demuestren evidencia de calidad técnica y valorar su uso o adaptación a través de ejercicios investigativos como el que se documenta en este artículo.

\subsection{Dimensiones para la evaluación de competencias de la Tutoría Académica}

Epstein y Hundert (2002 en Gutiérrez, 2005) definen la competencia profesional como "el uso habitual y juicioso de comunicación, conocimiento, habilidades técnicas, razonamiento clínico, emociones, valores y reflexión en la práctica cotidiana para el beneficio del individuo y la comunidad a los que se está ofreciendo un servicio" (p. 255).

El concepto anterior de competencia hace alusión a diversas áreas a desarrollar por el profesional, desde aspectos relacionales, técnicos, cognitivos y afectivos; para este estudio, nos acotaremos a las competencias que requiere el tutor para desarrollar su práctica tutorial. Las competencias del tutor, así como las competencias docentes, pueden ser clasificadas en tres dimensiones: previsión, conducción y valoración del impacto del proceso (García et al., 2008). Estas dimensiones son abordadas también en el método de este artículo.

García y otros (2016) consideraron en relación a la primera dimensión, "Previsión del proceso de tutoría” la competencia de planificar el proceso de tutoría, a lo cual Van (2000 en Álvarez, 2013) indica que la "labor de acompañamiento y guía que realiza el profesor tutor no puede afrontarse de un modo espontáneo, sino que requiere de una organización y planificación” (p. 82).

Respecto a la dimensión "conducción del proceso de tutoría" se abordan competencias relacionadas con la gestión, el seguimiento y la comunicación (García et al., 2016); las cuales corresponden a atributos interpersonales que el tutor debe de desarrollar. La disponibilidad, las habilidades de comunicación y de socialización le permitirán tener facilidad del para relacionarse, comunicarse, comprender y empatizar con sus tutorados (Berger, 1990; Fagenson-Eland, Marks y Amendola, 1997; Hartung, 1995; Maloney, 1999, citado por De la Cruz, Chehaybares y Abreu, 2011).

La última dimensión, titulada "Valoración del impacto del proceso de tutoría" aborda las competencias de valorar el efecto de los procesos de tutoría individual y grupal y la evaluación de la tutoría (García et al., 2016); dichas competencias permiten comprobar si los tutorados están satisfechos con el servicio y atención recibida, así como el impacto que este acompañamiento tiene en su aprendizaje; esto con la finalidad mejorar el plan de acción diseñado (Gómez, 2012). 


\section{Método}

El tipo de estudio fue cuantitativo, de alcance exploratorio y buscó identificar las propiedades métricas de un cuestionario aplicado en el contexto de una Institución de nivel superior. La muestra de estudio se conformó por 144 estudiantes inscritos en la Licenciatura en Educación Primaria. Más del 50\% fueron mujeres, en un rango de edad entre 20 y 22 años. Se consideró el $98 \%$ de representatividad, particularmente, del quinto semestre; la selección intencional obedeció al ciclo en el que el estudiante es acompañado por un tutor bajo el Modelo de Tutorías Académicas de ANUIES.

El cuestionario aplicado es producto de un trabajo de investigación (García et al., 2016); para la construcción del cuestionario se recurrió a la revisión de literatura nacional e internacional sobre tutorías académicas relacionadas con el modelo que plantea ANUIES: el de acompañamiento de estudiantes. Se destacan los siguientes autores: Álvarez (2002), Álvarez y Bisquerra (1996), Boza y otros (2005), Romo (2011), Molina y Amalia (2012), Rodríguez Espinar (2004), Watts y Esbroeck (2000), Astaiza (2008), Soto, Procel y Martínez (2008), Canales (s.f); Castillo, Polanco y Torreo (2009), Mogollón (2006), Castro (2012), Saavedra, Alatorre y Tremillo (2015), e Hidalgo, Cárdenas y Rodríguez (2013).

El cuestionario aplicado es una versión reducida del original, que se integraba por 80 reactivos, y que fue trabajado bajo la metodología propuesta por Jornet, González-Such y Perales (2012) para la optimización de la medida de constructos complejos; el proceso metodológico seguido para la optimización del instrumento se conformó por cuatro fases: 1) exploración del comportamiento inicial de los ítems a partir del análisis de varianza o escasa variabilidad; 2) análisis de confiabilidad y discriminación mediante análisis de correlación punto-biseral $<0,30$; 3) estimación de la puntuación total y exploración de su calidad a través del indicador punto-biseral $<0,30$, y alfa de Cronbach $<0,60 ;$ y 4) análisis de validez de constructo vía análisis de cargas factoriales $<0,30$ mediante componentes principales y extracción varimax. El instrumento en su versión depurada consta de 30 reactivos con una escala Likert que va desde totalmente en desacuerdo (TE), en desacuerdo (ED), de acuerdo (DA) hasta totalmente de acuerdo (TA) de respuesta a cada uno de ellos, los 30 reactivos se estructuran por las dimensiones que se muestran en el cuadro 2 .

El procedimiento de aplicación se realizó bajo la modalidad presencial, se aseguró que los participantes cumplieran los requisitos de inclusión necesarios en el estudio, el instrumento se aplicó en papel, asistido por un profesor en cada uno de los grupos de estudiantes seleccionados. El tratamiento de los datos de tipo descriptivo se hizo con el programa SPSS, versión 22. 
Cuadro 2. Dimensiones del Modelo de evaluación de competencias de la tutoría académica

\begin{tabular}{|c|c|c|c|}
\hline DIMENSIÓN & ÍTEMS & COMPETENCIA & DESCRIPCIÓN \\
\hline $\begin{array}{l}\text { Previsión del } \\
\text { proceso de } \\
\text { tutoría }\end{array}$ & $1-4$ & $\begin{array}{c}\text { Planificar el proceso de } \\
\text { tutoría }\end{array}$ & $\begin{array}{l}\text { Integra las metas institucionales con } \\
\text { las metas personales del alumno, } \\
\text { tomando en cuenta sus antecedentes } \\
\text { académicos, sociodemográficos, así } \\
\text { como sus expectativas. Incluye } \\
\text { objetivos, tareas, estrategias, tiempo, } \\
\text { recursos y compromisos tutor- } \\
\text { tutorado(s) a partir del programa } \\
\text { educativo, considerando las } \\
\text { condiciones específicas de la } \\
\text { institución (el referente normativo, } \\
\text { académico y administrativo). }\end{array}$ \\
\hline \multirow{3}{*}{$\begin{array}{l}\text { Conducción del } \\
\text { proceso de } \\
\text { tutoría }\end{array}$} & $5-11$ & $\begin{array}{c}\text { Gestionar el proceso de } \\
\text { tutoría }\end{array}$ & $\begin{array}{l}\text { Involucra acciones de apoyo integral a } \\
\text { los planes de tutoría en los tiempos } \\
\text { indicados por la institución, creando } \\
\text { un clima empático y de respeto, } \\
\text { propiciando el desarrollo personal y } \\
\text { profesional de los tutorados. }\end{array}$ \\
\hline & $13-18$ & $\begin{array}{c}\text { Dar seguimiento, } \\
\text { ajustar y monitorear las } \\
\text { acciones desarrolladas } \\
\text { en la tutoría }\end{array}$ & $\begin{array}{l}\text { Considera diversas modalidades de } \\
\text { retroalimentación a la formación de } \\
\text { los tutorados y estrategias de } \\
\text { monitoreo de la tutoría. }\end{array}$ \\
\hline & $19-23$ & $\begin{array}{l}\text { Utilizar estrategias } \\
\text { adecuadas de } \\
\text { comunicación para } \\
\text { realizar el trabajo de } \\
\text { tutoría }\end{array}$ & $\begin{array}{l}\text { Considera la comunicación asertiva de } \\
\text { ideas, conocimientos y emociones para } \\
\text { generar confianza y estar en sintonía } \\
\text { con los tutorados, así como la } \\
\text { promoción del pensamiento crítico y } \\
\text { reflexivo, en un clima de respeto y } \\
\text { confidencialidad. }\end{array}$ \\
\hline \multirow{2}{*}{$\begin{array}{l}\text { Valoración del } \\
\text { impacto del } \\
\text { proceso de } \\
\text { tutoría }\end{array}$} & \multirow[t]{2}{*}{$24-30$} & $\begin{array}{l}\text { Valorar el efecto de los } \\
\text { procesos de tutoría } \\
\text { individual y grupal }\end{array}$ & $\begin{array}{l}\text { Considera los logros alcanzados por la } \\
\text { tutoría, concertando las metas } \\
\text { formativas del alumno con las metas } \\
\text { institucionales. }\end{array}$ \\
\hline & & Evaluación de la tutoría & $\begin{array}{l}\text { Considera la forma en que el alumno } \\
\text { participa en el proceso de evaluación } \\
\text { de la tutoría. }\end{array}$ \\
\hline
\end{tabular}

Fuente: García y otros (2016).

\subsection{Proceso de análisis de propiedades métricas}

Para efectos de contar con evidencias de validez de constructo, se admite la premisa de que los ítems no comparten estructura de categorías uniformes entre ellos. La versión original del instrumento registró un índice de consistencia interna Alfa de Cronbach de 0,985. La versión optimizada registró consistencia interna Alfa de Cronbach $\alpha=0,967$. De igual forma, la correlación entre la puntuación total de la versión inicial y la versión optimizada es 0,987. Por ello, se adopta la técnica Rating Scale Model (RSM) de RaschAndrich (1978), dado que se asume que cada reactivo tiene su propia estructura de categorías y distancias. Lo anterior, teniendo como referencia que este tipo de procedimientos son los más adecuados para instrumentos en vías de consolidación y por ende, no se tiene evidencia uniforme compartida entre reactivos (González-Montesinos, 2008). Es el modelo de contraste adecuado cuando los ítems tienen patrones de respuesta en categorías ordenadas (Likert). El modelo Rasch-Andrich se desarrolló para estimar 
las propiedades de ítems politómicos con categorías de respuesta definidas. El modelo se define por:

$$
p_{i x}^{*}(\theta)=\frac{\exp \left[\alpha_{i}\left(\theta-\beta_{i j}\right)\right]}{1+\exp \left[\alpha_{i}\left(\theta-\beta_{i j}\right)\right]}
$$

Lo que representa la probabilidad de que la respuesta $x$ de una persona $s$ al ítem $i$ quede dentro o sobre el umbral de una categoría dada $\left(\mathrm{j}=1 . \mathrm{m}_{i}\right)$ condicionada por el nivel de rasgo $\theta$ (actitud, opinión, percepción) del sujeto. Y donde las probabilidades de que la persona se ubique dentro de una categoría específica se obtienen por:

$$
p_{i x}=p_{i x}^{*}(\theta)-p_{i(x+1)}^{*}(\theta)
$$

Como puede verse, en este tipo de ítems el modelo de contraste se modifica para incorporar un parámetro de dificultad a los umbrales de cambio de la categoría de respuesta $\mathrm{j}$ con respecto a la siguiente $\mathrm{j}+1$. En estos casos los criterios de bondad de ajuste interno y externo se aplican de la misma forma que en los ítems binarios. Así, tanto los ítems politómicos como los umbrales de paso de categoría a categoría deben mantenerse en el intervalo de 0,50 a 1,50 en ajuste interno y externo. El análisis se implementó con el programa WINSTEPS V.3.70.1 (Linacre, 2010).

\section{Resultados}

El proceso de validación llevado a cabo en el estudio permitió por un lado, afinar los reactivos de mayor calidad para la descripción oportuna de la función del tutor en el proceso de acompañamiento docente, por otro lado, mostró las propiedades psicométricas de unidimensionalidad del instrumento. Los hallazgos descriptivos de la aplicación del instrumento resaltaron la dimensión de conducción del proceso de tutoría; los respondentes ponderaron más del $50 \%$ de sus respuestas en la opción Totalmente de acuerdo; reactivos como Mi tutor me brindó confianza para expresar mis emociones $60 \%, \mathrm{Mi}$ tutor me hizo recomendaciones en relación a mi desempeño académico (70\%), Mi tutor estableció una comunicación cercana que facilitó mi entendimiento mutuo (64\%), Mi tutor me dedicó el tiempo necesario en las tutorías (78\%), Mi tutor me aseguró el trato confidencial de la información que yo proporcionara durante la tutoría (78\%) y Mi tutor utilizó algún medio electrónico como redes sociales, blogs, correo electrónico, plataformas, entre otros para facilitarme información relevante y de interés (85\%). La dimensión de menor aglutinación de respuestas fue Previsión de la tutoría, reactivos como Mi tutor me proporcionó información sobre Normatividad Universitaria (39\%) y Aproveche los servicios y recursos universitarios sugeridos por mi tutor para solventar mis necesidades particulares (43\%).

Los resultados correspondientes al análisis de reactivos Rasch-Andrich (1978) mostró evidencia de ajuste del modelo del instrumento (ver cuadro 3). En cuanto a los estadísticos por reactivo, se encontró que, en los estadígrafos de bondad de ajuste identificados en INFIT MSNQ y OUTFIT MSQN los reactivos se alinean a la expectativa del modelo métrico Rasch, ajustándose dentro del umbral esperado por el modelo, en el rango de .50 a 1.50. Los reactivos de menor MNSQ fueron: R1, R 21, R 7, y R 14, los de mayor puntaje R5, R9, R 25, R 21 y R 24. 
Cuadro 3. Estadígrafos de bondad de ajuste por reactivos

\begin{tabular}{|c|c|c|c|c|c|c|c|c|c|c|}
\hline \multirow{2}{*}{$\begin{array}{l}\text { NUMERACIÓN } \\
\text { DE ENTRADA }\end{array}$} & \multirow{2}{*}{$\begin{array}{l}\text { PUNTAJe } \\
\text { TOTAL }\end{array}$} & \multirow{2}{*}{$\begin{array}{c}\text { CUENTA } \\
\text { TOTAL }\end{array}$} & \multirow{2}{*}{ MEDIDA } & \multirow{2}{*}{$\begin{array}{c}\text { S.E } \\
\text { MOD }\end{array}$} & \multicolumn{2}{|c|}{ INFIT } & \multicolumn{2}{|c|}{ OUTFIT } & \multirow{2}{*}{$\begin{array}{c}\text { ITEM } \\
\text { G }\end{array}$} & \\
\hline & & & & & MNSQ & ZEMP & MNSQ & ZEMP & & \\
\hline 24 & 109 & 144 & 1,53 & , O4 & 1,14 & 1,3 & $-2,3$ &, 16 & $\mathrm{R} 24$ & $\mathrm{O}$ \\
\hline 2 & 112 & 141 & 1,50 &, 04 & 1,12 & 1,2 &,- 11 &, 18 & $\mathrm{R} 2$ & $\mathrm{O}$ \\
\hline 3 & 119 & 144 & 1,47 & ,03 & 1,10 & 1,4 &,- 01 & ,23 & $\mathrm{R} 3$ & $\mathrm{O}$ \\
\hline 15 & 138 & 142 & 1,16 & ,O3 & 1,13 & 1,3 &,- 07 &, 22 & $\mathrm{R} 15$ & $\mathrm{O}$ \\
\hline 4 & 145 & 140 & 1,09 &, 02 & ,98 &,- 4 & ,32 & ,29 & $\mathrm{R} 4$ & $\mathrm{O}$ \\
\hline 8 & 156 & 144 & 60 & ,02 & 1,03 &, 5 &, 22 &, 27 & $\mathrm{R} 8$ & $\mathrm{O}$ \\
\hline 30 & 159 & 140 &, 51 & ,02 &, 97 & 4 & ,32 &, 27 & R30 & $\mathrm{O}$ \\
\hline 26 & 180 & 135 &, 50 &, 02 & 1,02 &,- 4 &, 24 &, 28 & $\mathrm{R} 26$ & $\mathrm{O}$ \\
\hline 27 & 188 & 136 & , 49 & ,03 & 1,08 &, 5 &, 16 & ,29 & $\mathrm{R} 27$ & $\mathrm{O}$ \\
\hline 28 & 192 & 144 & 47 & ,03 & 1,09 & 1,2 &, 10 & ,26 & $\mathrm{R} 28$ & $\mathrm{O}$ \\
\hline 6 & 200 & 142 &, 47 & ,O2 & 1,10 & 1,3 &,- 04 &, 28 & $\mathrm{R} 6$ & $\mathrm{O}$ \\
\hline 9 & 209 & 142 &, 42 & ,O2 & 1,20 & 3,5 &, 10 &, 27 & $\mathrm{R} 9$ & $\mathrm{O}$ \\
\hline 5 & 216 & 141 & ,39 & ,03 & 1,26 &,- 3 &, 36 &, 22 & $\mathrm{R} 5$ & $\mathrm{O}$ \\
\hline 25 & 229 & 142 &,$- \mathrm{O} 2$ & ,03 & 1,12 &,- 2 &, 32 &, 22 & $\mathrm{R} 25$ & $\mathrm{O}$ \\
\hline 12 & 258 & 141 &,- 10 & ,02 & ,96 &,- 5 &, 35 &, 19 & $\mathrm{R} 12$ & $\mathrm{O}$ \\
\hline 20 & 278 & 140 &,- 28 & ,O3 & ,98 & ,3 &, 25 &, 18 & $\mathrm{R} 20$ & $\mathrm{O}$ \\
\hline 18 & 279 & 143 &,- 30 & ,03 &, 94 &,- 1 & ,26 &, 19 & $\mathrm{R} 18$ & $\mathrm{O}$ \\
\hline 23 & 283 & 143 &,- 30 & ,03 & 1,03 &,- 4 & ,33 &, 18 & $\mathrm{R} 23$ & $\mathrm{O}$ \\
\hline 14 & 287 & 142 &,- 34 & ,O2 & 1,05 &,- 2 &, 31 &, 19 & $\mathrm{R} 14$ & $\mathrm{O}$ \\
\hline 13 & 295 & 141 &,- 38 &, $\mathrm{O} 2$ & ,95 &,- 7 & ,32 &, 18 & $\mathrm{R} 13$ & $\mathrm{O}$ \\
\hline 17 & 299 & 144 &,- 40 & ,03 & ,96 &,- 4 &, 34 &, 19 & $\mathrm{R} 13$ & $\mathrm{O}$ \\
\hline 16 & 233 & 142 &,- 42 & ,03 & ,98 & -4 & ,38 &, 18 & $\mathrm{R} 17$ & $\mathrm{O}$ \\
\hline 14 & 246 & 143 &,- 45 & ,02 & ,93 &,- 5 & ,32 &, 17 & $\mathrm{R} 16$ & $\mathrm{O}$ \\
\hline 1 & 275 & 140 &,- 49 & ,03 &, 91 &,- 4 &, 37 &, 18 & $\mathrm{R}_{14}$ & $\mathrm{O}$ \\
\hline 10 & 284 & 140 &,- 70 & ,03 & ,95 &,- 1 &, 27 &, 14 & $\mathrm{R} 1$ & $\mathrm{O}$ \\
\hline 29 & 297 & 141 &,- 74 & ,04 &, 95 &,- 1 &, 23 &, 14 & $\mathrm{R} 10$ & $\mathrm{O}$ \\
\hline 21 & 311 & 141 &,- 93 & , O4 &, 92 &,- 4 &, 32 &, 15 & $\mathrm{R} 21$ & $\mathrm{O}$ \\
\hline 7 & 325 & 140 &,- 98 & ,O4 &, 92 & $-, 1,0$ & ,39 &, 15 & $\mathrm{R} 7$ & $\mathrm{O}$ \\
\hline 11 & 306 & 141 & $-1,00$ & ,O4 &, 97 &, 2 &, 25 &, 12 & $\mathrm{R} 11$ & $\mathrm{O}$ \\
\hline 22 & 309 & 141 & $-1, \mathrm{OO}$ & ,O4 &, 96 &, 10 &, 23 &, 10 & $\mathrm{R} 22$ & $\mathrm{O}$ \\
\hline $\begin{array}{c}\text { MEAN } \\
\text { (S.D.) }\end{array}$ & $\begin{array}{c}230,256 \\
(302,2)\end{array}$ & $\begin{array}{l}143,0 \\
(2,3)\end{array}$ & $\begin{array}{c}, \mathrm{OO} \\
(, 50)\end{array}$ & $\begin{array}{c}\text {,03 } \\
(, 01)\end{array}$ & $\begin{array}{l}1,00 \\
(, 08)\end{array}$ & $\begin{array}{c}, 2 \\
(1,0)\end{array}$ & $\begin{array}{l}1,01 \\
(, 10)\end{array}$ & $\begin{array}{c}2 \\
(1,0)\end{array}$ & & \\
\hline
\end{tabular}

Fuente: Elaboración propia.

Los niveles de dificultad de los reactivos calibrados se muestran en el apartado de MEASURE: van desde 1,54 arriba de la media, hasta $-1,0$ lógitos por debajo de la media, cabe resaltar que este grupo de respondentes no excede del rango -2 a +2 lógitos. En los resultados obtenidos sobre el comportamiento de los reactivos de acuerdo a sus categorías de respuesta, se encontró que los valores de ajuste interno (INFIT) y externo (OUTFIT) se comportan cercanos a 1 , y en todos los casos se mantienen dentro del intervalo de 0,50 a 1,50. Lo anterior demuestra apego al criterio de buen ajuste en reactivos politómicos de percepción, tal como se muestra en la figura 1.

Por otra parte, las categorías de respuesta al interior de cada uno de los 30 reactivos presentan el mismo comportamiento, ajustándose a las expectativas del modelo de contraste RSM. Como puede observarse, la aplicación puntual de los criterios de bondad de ajuste de los reactivos y de las categorías de respuesta graduada al interior de ellos se alinea de manera productiva a las expectativas del modelo (ver cuadros 4 y 5). Los respondientes expresan sus grados de percepción con respecto a las 30 afirmaciones del instrumento. 


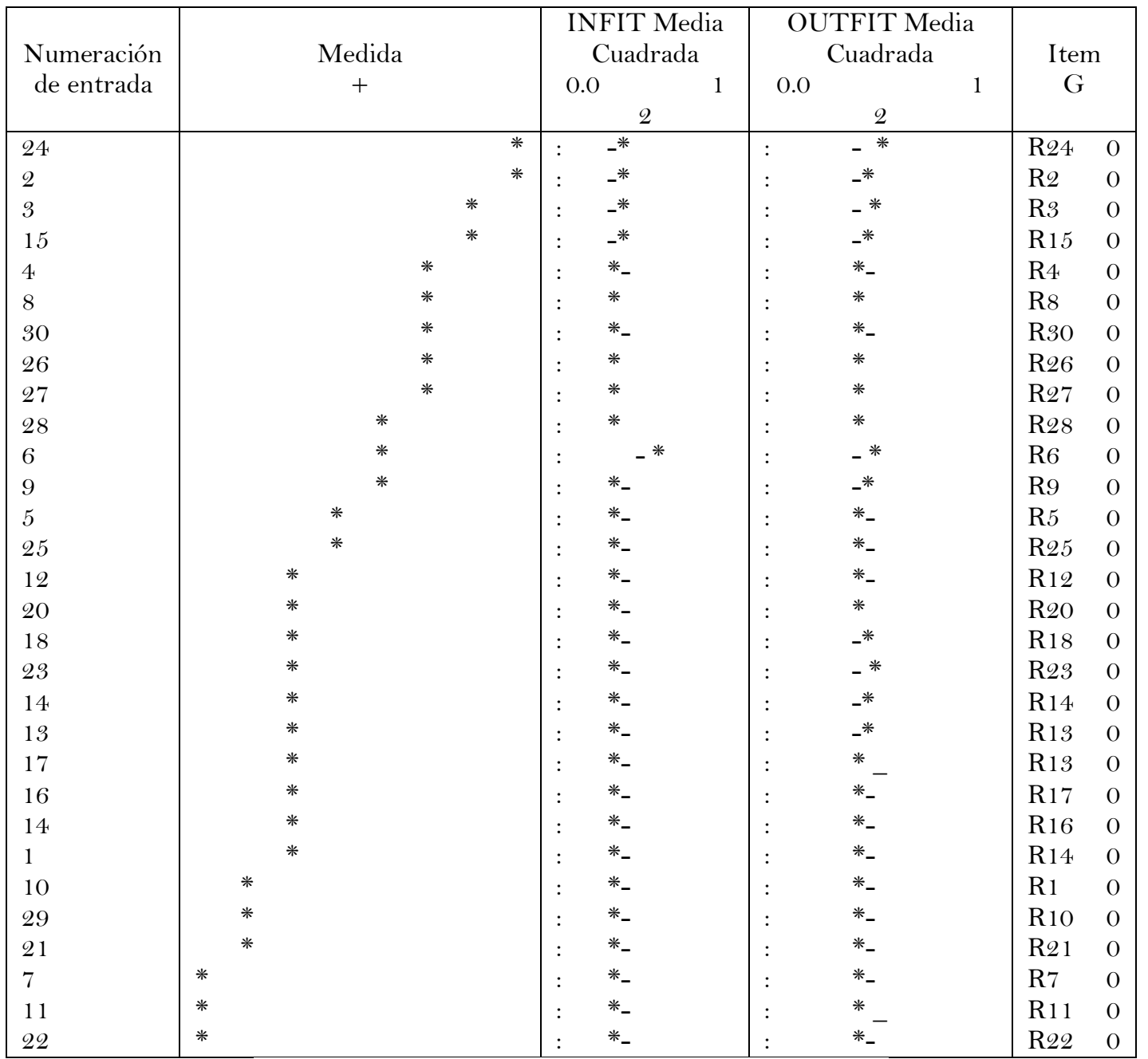

Figura 1. Gráfico de bondad de ajuste por reactivos

Fuente: Elaboración propia.

Cuadro 4. Ajuste de reactivos principales por categoría de respuesta

\begin{tabular}{|c|c|c|c|c|c|c|c|c|c|}
\hline $\begin{array}{l}\text { ETIQUETA } \\
\text { POR } \\
\text { CATEGORIA }\end{array}$ & Puntaje & $\begin{array}{c}\text { DATOS } \\
\text { OBSERVADOS }\end{array}$ & $\begin{array}{l}\text { MUESTRA } \\
\text { PROMEDIO }\end{array}$ & $\begin{array}{l}\text { MUESTRA } \\
\text { ESPERADA }\end{array}$ & $\begin{array}{l}\text { IN } \\
\text { OU? }\end{array}$ & $\begin{array}{l}\text { IT } \\
\text { FIT }\end{array}$ & $\begin{array}{l}\text { ESTRUCTURA } \\
\text { CALIBRADA }\end{array}$ & $\begin{array}{l}\text { CATEGORÍA } \\
\text { Y MEDIDA }\end{array}$ & \\
\hline 1 & 1 & 9 & 7 & 0,12 & 1,02 & 1,15 & NONE & $-2,02$ & $\begin{array}{c}1 \text { Tot. en } \\
\text { desacuerdo }\end{array}$ \\
\hline 2 & 2 & 22 & 9 & 0,17 & 0,98 & 0,93 & $-0,53$ & $-1,47$ & 2 Desacuerdo \\
\hline 3 & 3 & 33 & 8 & 0,13 & 0,95 & 0,91 & 0,58 & $-0,81$ & 3 Indeciso \\
\hline 4 & 4 & 64 & 6 & 0,16 & 0,88 & 0,83 & $-1,03$ & $-0,09$ & 4 De acuerdo \\
\hline 5 & 5 & 14 & 12 & 0,14 & 0,92 & 0,97 & 1 & $-1,47$ & $\begin{array}{l}5 \text { Tot. de } \\
\text { acuerdo }\end{array}$ \\
\hline Missing & 2 & 0,2 & & & & & & & \\
\hline
\end{tabular}

Nota: Medida de dificultad del Îtem de -.64 Agregado a la medida.

Fuente: Elaboración propia. 
Cuadro 5. Ajuste de las categorías de respuesta

\begin{tabular}{|c|c|c|c|c|c|c|c|c|}
\hline $\begin{array}{l}\text { NUMERACIÓN } \\
\text { DE ENTRADA }\end{array}$ & Datos & $\begin{array}{l}\text { VALOR DE } \\
\text { PUNTAJE }\end{array}$ & $\begin{array}{c}\text { CONTEO } \\
\text { DE DATOS } \\
\%\end{array}$ & $\begin{array}{c}\text { PROMEDIO } \\
\text { DE } \\
\text { HABILIDAD }\end{array}$ & $\begin{array}{c}\text { S.E. } \\
\text { MEDIA } \\
\text { MNSQ }\end{array}$ & $\begin{array}{c}\text { OUTFIT } \\
\text { MNSQ }\end{array}$ & $\begin{array}{c}\text { PTMEA } \\
\text { CORR. }\end{array}$ & ITEM \\
\hline \multirow[t]{5}{*}{24} & 5 & 5 & 89 & .54 & & 1,1 & ,30 & $\mathrm{R} 24$ \\
\hline & 4 & 4 & 31 & 41 & , O1 & 1,2 &,- 25 & \\
\hline & 3 & 3 & 22 & ,30 & ,03 & 1,2 &,- 06 & \\
\hline & 2 & 2 & 2 & , 49 & ,05 & 1,5 &,- 09 & \\
\hline & 1 & 1 & $\mathrm{O}$ & ,66 & ,07 & 1,2 & ,01 & \\
\hline \multirow[t]{5}{*}{2} & 5 & 5 & 92 &, 51 & , 01 & 1,1 &, 20 & $\mathrm{R} 2$ \\
\hline & 4 & 4 & 39 & , 41 & , O 1 & 1,3 &,- 21 & \\
\hline & 3 & 3 & 10 & , 44 & ,03 & 1,2 & ,OO & \\
\hline & 2 & 2 & 2 &, 54 & ,04 & 1,3 &,- 03 & \\
\hline & 1 & 1 & 1 &, 54 & ,06 & 1,2 & ,04 & \\
\hline \multirow[t]{5}{*}{3} & 5 & 5 & 100 &, 50 & , O1 & 1,1 &, 14 & $\mathrm{R} 3$ \\
\hline & 4 & 4 & 25 & ,42 & , O1 & 1,3 &,- 17 & \\
\hline & 3 & 3 & 17 & 42 & , 02 & 1,2 & ,04 & \\
\hline & 2 & 2 & 1 &, 51 & ,03 & 1,3 &, 10 & \\
\hline & 1 & 1 & $\mathrm{O}$ &, 58 & ,06 & 1,1 & ,03 & \\
\hline \multirow[t]{5}{*}{15} & 5 & 5 & 78 &, 52 & , O 1 & 1,0 &, 16 & $\mathrm{R} 15$ \\
\hline & 4 & 4 & 35 & ,43 & , 01 & 1,1 &,- 17 & \\
\hline & 3 & 3 & 30 & ,49 & ,04 & 1,6 & ,04 & \\
\hline & 2 & 2 & 1 & ,35 & ,04 & 1,2 &,- 12 & \\
\hline & 1 & 1 & $\mathrm{O}$ & ,36 & ,06 & 1,1 & ,07 & \\
\hline \multirow[t]{5}{*}{4} & 5 & 5 & 98 &, 52 & , 01 & 1,1 &,- 08 & $\mathrm{R} 4$ \\
\hline & 4 & 4 & 22 & , 43 & , 01 &, 8 &,- 24 & \\
\hline & 3 & 3 & 17 & 49 & , 02 & 1,1 &,- 03 & \\
\hline & 2 & 2 & 6 & ,35 & , 02 & 1,2 & , 01 & \\
\hline & 1 & 1 & 1 &, 55 & ,O2 & ,9 & ,37 & \\
\hline \multirow[t]{5}{*}{8} & 5 & 5 & 56 & , 44 & ,02 & 1,2 & ,03 & $\mathrm{R} 8$ \\
\hline & 4 & 4 & 34 & ,39 & , 01 &, 9 &,- 03 & \\
\hline & 3 & 3 & 32 & 46 & , 01 & 1,0 &,- 19 & \\
\hline & 2 & 2 & 21 & 48 & , 01 & 1,2 & ,02 & \\
\hline & 1 & 1 & 1 &, 57 & ,02 & ,9 &,- 07 & \\
\hline
\end{tabular}

Nota: Medida de dificultad del Îtem de -.74 Agregado a la medida.

Fuente: Elaboración propia.

\section{Discusión y conclusiones}

El estudio de la importancia del proceso de tutoría en la formación profesional de los estudiantes de educación superior resulta interesante y trascendente para consolidar procesos de calidad educativa, no sólo por la riqueza que aporta al conocimiento, sino por el estudio, a través de las dimensiones que se derivan de la operación del modelo de tutorías en la educación superior (ANUIES, 2001). Hablar de acción tutorial tiende a la asociación mecánica entre el proceso de acompañamiento hacia el alumno y el papel que desempeña el tutor en los aspectos cognitivos y afectivos de su aprendizaje.

El instrumento sometido a estudio ha supuesto gran esfuerzo de clarificación y consolidación conceptual del constructo estudiado, así como ha fortalecido ensolidado una directriz en torno al estudio empírico en actitudes orientadas hacia la acción tutorial. La investigación para la que fue diseñado se encuentra actualmente en curso. Por ello, los hallazgos que ha proporcionado este análisis tienen implicaciones métricas importantes para la acumulación de evidencias de confiabilidad y validez de constructo de un instrumento para estudiantes de la Escuela Normal en el contexto mexicano. Un aspecto de interés en el presente estudio está relacionado con el ajuste de los datos al modelo. Al respecto, se observa que el cuestionario muestra un nivel de ajuste al modelo, 
por ítems y por categorías de respuesta. Esto se ve indicado en el ajuste de los puntajes alrededor de la media y de los extremos (véase los cuadros 2 y 3), que se encuentra dentro de los parámetros establecidos por el modelo. Debido a lo anterior, se puede afirmar que este instrumento mide un factor dominante, que es la acción tutorial, por lo que este instrumento resulta unidimensional (Muñiz, 1997).

Los resultados en las tres dimensiones que conforman el Modelo de evaluación de competencias de la tutoría académica, presentan porcentajes bastante altos en las escalas de totalmente en desacuerdo y en desacuerdo. Por ello, se concluye que la Escuela Normal en la que se llevó acabo el estudio, requiere de un trabajo de tutoría que implemente lo establecido en el modelo tutorial propuesto por la ANUIES, dando un seguimiento constante para evaluar el proceso de la tutoría académica.

La dimensión de mayor ponderación por parte de los participantes fue Conducción del proceso de tutoría En esta dimensión se describe la percepción de los jóvenes hacia el interés y disposición personal que muestra el docente-tutor en el establecimiento de ambientes favorables para la acción tutorial, son considerados como elementos importantes para su permanencia y el logro de un buen desarrollo personal y académico; resaltaron aspectos de confianza, comunicación y seguimiento por parte del tutor, lo cual se asemeja al estudio presentado por Conejeros, Rojas y Segura (2010), quienes encontraron que una relación de confianza propicia un clima apto para el dialogo, así como para la resolución de conflictos y problemas en los estudiantes lo cual enriquece el proceso de tutoría académica.

Otro aspecto de los resultados descriptivos del instrumento fue la ponderación al reactivo Mi tutor se interesó por conocer aspectos de mi vida personal, familiar y social, el 49\% respondió totalmente en desacuerdo, lo cual se relaciona con el estudio realizado por Osuna y Díaz (2015), encontraron que más del 70 \% de los alumnos indicó que a sus profesores no les interesaba conocer sus aspectos familiares o de su vida personal. Se podría concluir que se privilegia el interés del tutor por situaciones de índole académica de los estudiantes sobre aspectos personales y familiares.

Resulta necesario, continuar con investigaciones a partir de estudios diagnósticos in situ, que cedan la voz a los estudiantes y a través de estas, indagar en los aspectos subjetivos de las tutorías, con la intención de identificar posibles áreas de intervención en pro de mejorar los procesos socio afectivos que posibiliten relaciones de cercanía y confianza entre maestros tutores y sus estudiantes (Boroel, Sánchez, Morales y Herníquez, 2018).

Los hallazgos también muestran evidencia de la necesidad de entender los tipos de tutorías que se desarrollan en la formación de estudiantes de Escuelas Normales, así como de establecer con claridad las funciones en cuanto al acompañamiento académico de los estudiantes en el desarrollo de sus trayectos formativos y el del desarrollo de su trabajo terminal e intervención en campo, evitando confusiones entre estos roles de tutoría. A reserva de que el presente cuestionario sea sujeto a procesos de análisis continuos que le permita contar con estructuras más simples, parsimoniosas y de fácil aplicación, los datos observados en torno al desarrollo de este instrumento a una muestra de estudiantes mexicanos de escuelas normales en el nivel superior, proporciona el soporte necesario para su utilización. Su adecuación con los marcos conceptuales prevalecientes, los índices de ajuste y discriminación registrados por sus ítems y los índices de confiabilidad observados, permiten realizar inferencias válidas y confiables, a partir de su aplicación a muestras similares de estudiantes. 
Finalmente, se considera importante realizar más exploraciones sobre el campo de la tutoría en las Escuelas Normales para entender sus realidades y, de esta forma, aportar al desarrollo de modelo de evaluación pertinentes y orientados a la mejora que, sin duda alguna, contribuyen al fortalecimiento de la formación de los estudiantes y de los tutores.

\section{Referencias}

Asociación Nacional de Universidades e Instituciones de Educación Superior. (2001). Programas Institucionales de Tutoría. Una propuesta de la ANUIES para su organización y funcionamiento en las instituciones de educación superior. Ciudad de México: ANUIES.

Asociación Nacional de Universidades e Instituciones de Educación Superior. (2010). La percepción del estudiante sobre la acción tutorial. Modelos para su evaluación. Ciudad de México: ANUIES.

Álvarez, M. y Bisquerra, R. (1996). Aproximación al concepto de orientación y tutoría. Madrid: Praxis.

Álvarez, P. (2002). La función tutorial en la universidad. Una apuesta por la mejora de la calidad de la enseñanza. Madrid: EOS Universitaria.

Álvarez, P. (2013). La tutoría como eje articulador del proceso de aprendizaje del alumnado universitario. Revista Qurriculum, 26, 73-87.

Álvarez, P. y González, M. C. (2009). Modelo comprensivo para la institucionalización de la orientación y la tutoría en la enseñanza universitaria. Revista Qurriculum, 22, 73-95.

Astaiza, N. (2008). El acompañamiento a los estudiantes en las instituciones de educación superior. Bogotá: SciELO.

Boza, A., Salas, M., Ipland, J., Aguaded, C., Fondon, M., Montescillo, M. y Méndez, J. (2005). Ser profesor, ser tutor. Orientación educativa para docentes. Madrid: Publidisa.

Boroel, B., Sánchez, J., Morales, K. y Henríquez, P. (2018). Educación exitosa para todos: la tutoría como proceso de acompañamiento escolar desde la mirada de la equidad educativa. Revista Fuentes, 20(2), 91 -104. https://doi.org/10.12795/revistafuentes.2017.v20.i2.06

Canales, E. (2010). El Perfil del tutor académico. Ciudad de México: Universidad del Estado de Hidalgo.

Castillo, S., Polanco, L. y Torreo, J. (2009). Tutoría en la enseñanza, la universidad y la empresa; Formación y práctica. Madrid: Pearson.

Castro, R. (2012). Manual de tutoría y plan de acción tutorial basado en competencias profesionales. Ciudad de México: Universidad Tecnológica de Chihuahua.

Conejeros, M., Rojas, J. y Segura, M. (2010) Confianza: un valor necesario y ausente en la educación chilena. Perfiles Educativos, 32(130), 30-46.

De la Cruz, G., Chehaybares, E. y Abreu, L. F. (2011). Tutoría en educación superior: una revisión analítica de la literatura. Revista de la Educación Superior, 1(157),190-209.

De la Cruz, G., Díaz-Barriga, F. y Abreu, L. F. (2010). La labor tutorial en los estudios de posgrado. Rúbricas para guiar su desempeño y evaluación. Perfiles Educativos, 32(130), 83102.

De la Cruz, G., Chehaybares, E. y Abreu, L. F. (2011). Tutoría en educación superior: una revisión analítica de la literatura. Revista de la Educación Superior, 1(157), 190-209. 
Diario Oficial de la Federación. (2012a). Acuerdo número 649 por el que se establece el Plan de Estudios para la Formación de Maestros de Educación Primaria. Recuperado de http://dof.gob.mx/nota_detalle.php?codigo=5264718\&fecha=20/08/2012

Diario Oficial de la Federación. (2012b). Acuerdo número 650 por el que se establece el Plan de Estudios para la Formación de Maestros de Educación Preescolar. Recuperado de http://dof.gob.mx/nota_detalle.php?codigo=5264719\&fecha=20/08/2012

Dirección General de Educación Superior para Profesionales de la Educación. (s.f). Planes de Estudio. Estrategias de apoyo para los estudiantes. Recuperado de https://www.dgespe.sep.gob.mx/web_old/reforma_curricular/planes/lepri/plan_de_estu dios/estrategias_apoyo_estudiantes

Gaitán, P. (2013). Hacia una definición de tutoría universitaria. DIDAC, 61, 4-9.

García, B., Loredo, J., Luna, E. y Rueda, M. (2008). Modelo de evaluación de competencias docentes para la educación media y superior. Revista Iberoamericana de Evaluación Educativa, 1(3), 96-108.

García, B., Ponce, S., García, M., Caso, J., Morales, C., Martínez, S., Serna, A. y Aceves, Y. (2016). Las competencias del tutor universitario: una aproximación a su definición desde la perspectiva teórica y de la experiencia de sus actores. Perfiles Educativos, 38(151), 104-122.

Gómez, M. E. (2012). La percepción de los estudiantes sobre el Programa de Tutoría Académica. Convergencia, 58, 209-233.

González-Montesinos, M. (2008). Análisis de reactivos con el Modelo Rasch. Ciudad de México: Manual Técnico del INEE.

Gutiérrez, O. (2005). Educación y entrenamiento basados en el concepto de competencia: Implicaciones para la acreditación de los programas de Psicología. Revista Mexicana de Psicología, 22, 253-270.

Hidalgo, J., Cárdenas, M. y Rodríguez, S. (2013). El tutor clínico. Una mirada de los estudiantes de Licenciatura de Enfermería y Obstetricia. Enfermería Universitaria, 10(3), 92-97.

Jiménez, A. J. y Villafuerte, M. M. (noviembre, 2011). Hacia la construcción del perfil del tutor en escuelas normales públicas del D.F.: Reflexiones desde la mirada de sus actores. Comunicación presentada en el XI Congreso Nacional de Investigación Educativa, Ciudad de México. Recuperado de: http://www.comie.org.mx/congreso/memoriaelectronica/v11/docs/area_15/1864.pdf

Jornet, J., González-Such, J. y Perales, M. (2012). Diseño de cuestionarios de contexto para la evaluación de sistemas educativos: optimización de la medida de constructos complejos. Bordón, 64(2), 89-110.

Linacre, J. (2010). Rasch-model computer programs. Chicago, IL: Electronic Publication.

Mapén, T. C., Pérez Cupil, M. y López Iglesia, A. A. (2016). Evaluación del programa de tutoría en la Escuela Normal de Educación Preescolar "Rosario María Gutiérrez Eskildsen". Perspectivas Docentes, 59, 13-18. https://doi.org/10.19136/pd.aOn59.1304

Minor, N., Castellanos, A. I. y Gómez, C. E. (noviembre, 2016). El programa de tutoría en la escuela normal rural: realidades de su implementación. Conunicación presentada en el 7 Encuentro Nacional de Tutoría, Guanajuato. Recuperado de: http://oa.ugto.mx/wp-content/uploads/2016/11/oa-rg-0000513.pdf

Mogollón, A. (2006). Éxito del tutor universitario. Revista Ciencias de la Educación, 1(27), 109-122.

Molina, A. (2012). Estado del arte sobre tutorías. Civilizar. Ciencias Sociales y Humanas, 12(22), 167-175. 
Muñiz, F. (1997). Introducción a la teoría de respuesta al ítem. Madrid: Editorial Piramide.

Osuna, C. y Díaz, K. (noviembre, 2015). Estudio sobre jóvenes desertores de la educación media superior en Baja California, México. Conmunicación presentada en el XIII Congreso Nacional de Investigación Educativa, Chihuahua.

Pérez Cusco, F. J., Martínez Clarés, P. y Martínez Juárez, M. (2015). Satisfacción del estudiante universitario con la tutoría. Diseño y validación de un instrumento de medida. Estudios Sobre Educación, 29, 81-102. https://doi.org/10.15581/004.29.81-101

Ponce, S., Aviña, I., Garcia, B. y Caso, J. (2018). Caracterización de los cuestionarios de evaluación de tutores universitarios. Aproximaciones a un diagnóstico nacional. Informe técnico de investigación. Ciudad de México: Universidad autónoma de Baja California.

Ramírez, R., Campos, R. y Rodríguez, R. (2013). El estudiante normalista en el programa de tutoría. Revista Iberoamericana para la Investigación y el Desarrollo Educativo, 10, 2-23.

Ramos, S., González, J. C. (2011). La tutoría y el aprendizaje. En S. C. Hernández y R. A. Andrade (Coords.), Aprendizaje y competencias en educación, visiones y reflexiones (pp. 221241). Ciudad de México: Universidad de Guadalajara.

Rasch-Andrich, G. (1978). Probabilistic models for some attainment and intelligence tests. Copenhague: Danmarks Pedagogiske Institut.

Redondo, S., Vale, P., Navarro, E. y Madrigal, A. M. (2012). Orientación educativa: fundamentos teóricos, modelos institucionales y nuevas perspectivas. Madrid: Ministerio de Educación.

Romo, A. (2010). La percepción del estudiante sobre la acción tutorial. Modelos para su evaluación. Ciudad de México: ANUIES.

Romo, A. (2011). La Tutoría. Una estrategia innovadora en el marco de los programas de atención a estudiantes. Ciudad de México: ANUIES.

Rodríguez Espinar, S. (2004). Manual de tutoría universitaria. Recursos para la acción. Madrid: Octaedro.

Saavedra, R., Alatorre, E. y Tremillo, R. (2015). Formación de tutores en las escuelas normales dentro de la reforma curricular del 2012. En M. Barrón, S. Torres y T. Muñoz (Coords.), Señalando rutas...que trazan el futuro. Programa institucional de tutorías (pp. 3-13). Ciudad de México: Universidad Autónoma de Coahuila.

Secretaría de Educación Pública. (2012). Lineamientos para organizar el proceso de titulación. Recuperado de

http://www.dgespe.sep.gob.mx/public/documentos_orientadores/lineamientos_para_org anizar_el_proceso_de_titulacion.pdf

Soto, J., Procel, A. y Martínez, N. (2008). Evaluación del Programa Institucional de Tutoría Académica (PITA) del Instituto Tecnológico Superior de Irapuato (ITESI) 2003-2007. Ciudad de México: Instituto Tecnológico Superior de Irapuato.

Universidad Autónoma de Baja California. (2012). Acuerdo que establece los Lineamientos generales para la operación de las Tutorías Académicas. Recuperado de http://www.uabc.mx/formacionbasica/documentos/Acuerdo_Tutorias.pdf

Valenzuela, F. y Herrera, I. (noviembre, 2016). La acción tutora en la formación del docente normalista. 7mo. Encuentro Nacional de Tutoría. Conunicación presentada en el 7 Encuentro Nacional de Tutoría, Guanajuato. Recuperado de: http://oa.ugto.mx/wp-content/uploads/2016/11/oa-rg-0000617.pdf 
Watts, A. y Esbroeck, R. (2000). New skills for new futures: a comparative review of higher education guidance and counselling services in the European Unión. International Journal for the Advancement of Counselling, 22, 173-187. https://doi.org/10.1023/A:1005653018941

Zazueta, M. y Herrera, L. (2008). Evaluación del desempeño del docente como tutor y la satisfacción de los tutorados en la Facultad de Odontología de la UAC-2008. Puebla de Zaragoza: Benemérita Universidad Autónoma de Puebla.

\section{Breve $\mathrm{Cv}$ de los autores}

\section{Salvador Ponce Ceballos}

Doctor en Educación. Profesor investigador de la Universidad Autónoma de Baja California. Es Perfil PRODEP; miembro del Cuerpo Académico Consolidado "formación, desarrollo y evaluación de actores educativos"; miembro del Consejo Mexicano de Investigación Educativa y del Consejo Técnico del Comité para la Evaluación de Programas de Pedagogía y Educación. Miembro del Sistema Nacional de Investigadores Nivel 1. Sus líneas de trabajo se ubican en en los campos de la formación docente, evaluación educativa, acompañamiento estudiantil y educación en línea. Actualmente es Coordinador General de Formación Profesional de la UABC. ORCID ID: https://orcid.org/0000-0003-0454-9853. Email: ponce@uabc.edu.mx

\section{Yaralin Aceves Villanueva}

Doctora en Educación. Profesora investigadora de la Universidad Autónoma de Baja California. Es Perfil PRODEP; miembro del Cuerpo Académico "Psicología Educativa y Desarrollo del Potencial Humano"; miembro del Consejo Mexicano de Investigación Educativa y de la Red Latinoamericana de Profesionales de la Orientación. Sus líneas de trabajo se ubican en en los campos de la orientación educativa, la tutoría y la evaluación del aprendizaje. Actualmente es Jefa del Departamento de Apoyo a la docência e Investigacion, Campus Mexicali de la UABC. ORCID ID: https://orcid.org/0000-00028175-2639. Email: yaralin@uabc.edu.mx

\section{Brenda Imelda Boroel Cervantes}

Doctora en Ciencias Educativas. Profesor Investigador de la Facultad de Ciencias Administrativas y Sociales - UABC. Perfil PRODEP, Miembro del Cuerpo Académico en Consolidación: Evaluación, Relaciones escolares y Salud. Miembro del grupo de Investigación GRIOCE. Miembro del Sistema Nacional de Investigadores Nivel C. Líneas de investigación: Evaluación del perfil profesional en educación superior, Equidad educativa y procesos de acompañamiento escolar. ORCID ID: https://orcid.org/oooo0002-2378-2901. Email: brenda.boroel@uabc.edu.mx 